\title{
Effects of resonant single-particle states on pairing correlations
}

\author{
Munetake Hasegawa ${ }^{1}$ and Kazunari Kaneko ${ }^{2}$ \\ ${ }^{1}$ Laboratory of Physics, Fukuoka Dental College, Fukuoka 814-0193, Japan \\ ${ }^{2}$ Department of Physics, Kyushu Sangyo University, Fukuoka 813-8503, Japan
}

(Dated: November 10, 2018)

\begin{abstract}
Effects of resonant single-particle (s.p.) states on the pairing correlations are investigated by an exact treatment of the pairing Hamiltonian on the Gamow shell model basis. We introduce the s.p. states with complex energies into the Richardson equations. The solution shows the property that the resonant s.p. states with large widths are less occupied. The importance of many-body correlations between bound and resonant prticle pairs is shown.
\end{abstract}

PACS numbers: 21.10.Dr, 21.60.Cs, 21.60.Ev

One of current topics is concerned with the microscopic structure of nuclei far from the $\beta$-stability line. In such nuclei, weak binding of nucleons is expected to cause exotic situations where resonant and continuum singleparticle (s.p.) states contribute considerably to nuclear properties even at low energy. The problem has been considered since an early time [1], and recent experimental study of drip-line nuclei has stimulated a new interest in it. The resonant and continuum s.p. states could enhance pairing correlations [2, 3] which are most important in nuclei once the Hartree-Fock (HF) or shell-model mean field is formed. They could affect binding energies of bound states and nuclear resonant states, and could play important roles in properties of these low-energy states. Recently, theoretical studies [4, 5, 6, 7] on the contributions from the resonant and continuum states have been reported. Refs. [4, 5] incorporated the effect of the resonant continuum or resonant states in the HF+BCS approximation. Refs. [6, 7] studied the effects of the resonant and continuum s.p. states on one pair state outside a core.

The purpose of this paper is to investigate the effects of the resonant s.p. states on many-body correlations in many particle systems (including correlations between bound and resonant particle pairs), on the shell-model basis. We treat the problem by exactly solving the pairing Hamiltonian including resonant s.p. states. Namely, we extend the Richardson equations [8] so as to include s.p. states of complex energies and solve them. The Richardson equations have a defect that the pairing interaction strength cannot be changed for bound and resonant s.p. states. Still, this approach is very useful for getting a general perspective of our subject.

We consider a system of Fermions in which the mean field and residual interaction are approximately represented by a one-body potential and the pairing force. We denote a Fermion by $c_{\nu \sigma}^{\dagger}(\sigma= \pm$ denote timereversal conjugate states) and a pair operator by $S_{\nu}^{\dagger}=$ $c_{\nu+}^{\dagger} c_{\nu-}^{\dagger}$. The eigenstates of our system are classified by the number of unpaired Fermions. The states with no unpaired Fermion are lower in energy than the others. We pay our attention only to such pair-correlated states composed of the basis states $S_{\mu}^{\dagger} S_{\nu}^{\dagger} \cdots|0\rangle$. In this subspace, the pairing Hamiltonian is written as
$H=\sum_{\nu} 2 \varepsilon_{\nu} N_{\nu}-G \sum_{\mu \nu} S_{\mu}^{\dagger} S_{\nu}$, where $\varepsilon_{\nu}$ denotes the s.p. energies and $G$ is the pairing force strength. The operator $N_{\nu}=S_{\nu}^{\dagger} S_{\nu}$ satisfies the relation $\left[N_{\nu}, S_{\nu}^{\dagger}\right]=S_{\nu}^{\dagger}$ from the Pauli principle $c_{\nu \sigma}^{\dagger} c_{\nu \sigma}^{\dagger}=0$, and counts the pair number in the level $\nu$. Richardson [8] proved that a pair-correlated eigenstate is expressed as $\prod_{k=1}^{N}\left\{\sum_{\nu} S_{\nu}^{\dagger} /\left(2 \varepsilon_{\nu}-z_{k}\right)\right\}|0\rangle$, using the roots $z_{k}$ of the coupled equations

$$
\sum_{\nu} \frac{1}{2 \varepsilon_{\nu}-z_{k}}=\frac{1}{G}+\sum_{k^{\prime}(\neq k)} \frac{2}{z_{k^{\prime}}-z_{k}} .
$$

Here, the number of the equations (10) is the number of pairs $N$. For an eigenstate, the total energy and occupation probability of the s.p. level $\nu$ are given by

$$
\begin{aligned}
& E=\langle H\rangle=\sum_{k=1}^{N} z_{k}, \\
& v_{\nu}^{2}=\left\langle N_{\nu}\right\rangle=-G^{2} \frac{d}{d G} \sum_{k=1}^{N} \frac{1}{2 \varepsilon_{\nu}-z_{k}} .
\end{aligned}
$$

Although Richardson [8] supposed $\varepsilon_{\nu}$ to be real number, his proof is not relevant to whether $\varepsilon_{\nu}$ are real or complex. We can therefore introduce resonant s.p. states with complex energies to our pairing problem by considering a Woods-Saxon potential. In compensation for this, however, the Hamiltonian stops being Hermitian. Even when the s.p. energies $\varepsilon_{\nu}$ are real, some of the roots $z_{k}$ are complex conjugate (the total energy of the system is real). If any of $\varepsilon_{\nu}$ are complex in Eqs. (10), all the roots $z_{k}$ are complex but not complex conjugate. If the resonant s.p. states participate in the pairing correlations, therefore, the total energies of the pair-correlated states including bound states become complex in general.

Let us first investigate basic properties of a general system which has resonant s.p. states. We consider a model that has a dozen s.p. levels with equal spacing $e$, where the lower eight levels are bound states and the upper four levels have the same resonant width $\gamma$,

$$
\varepsilon_{\nu}= \begin{cases}(\nu-8.5) e & \text { for } \nu=1,2, \cdots 8 \\ (\nu-8.5) e-i(\gamma / 2) e & \text { for } \nu=9,10,11,12 .\end{cases}
$$

We use the energy unit $e=1$ (also for $G$ and $\gamma$ ) in the following pages. 
When the pair number $N$ is small, the Fermi surface lies deeply below the resonant s.p. levels. The resonant states can be neglected as long as the pairing force is not very strong. This is the case of ordinary treatment for the nuclear pairing problem. Calculated results for the $N=4$ system are summarized as follows. The system gets additional energy gain when the resonant levels are taken into account and the energy gain increases more rapidly than that in case of no resonant levels as the pairing force becomes stronger. The total energy becomes complex, namely, eigenstates including the ground state have finite resonant width when the resonant s.p. states contribute to the pairing correlations, as mentioned above. Interestingly, the ground state has pair decay width larger than low-energy excited states.

We are interested in the situation of weak binding of nuclei where the Fermi surface lies near the resonant s.p. levels. Fig. 1 shows the effects of the resonant s.p. levels on the $N=8$ system with $\gamma / 2=0.5$, in which the Fermi surface is between the bound and resonant levels. If we do not take account of the resonant levels, there is no configuration mixing like the closed shell $\left(v_{\nu}^{2}=1\right.$ for $\nu \leq 8$ ) and hence the energy gain due to the pairing correlations increases monotonously depending on $G$. Fig. 1 indicates more rapid increase of the energy gain when the resonant s.p. states are taken into account. The imaginary part of the total energy shown in the lower graph of Fig. 1 displays interesting behavior. When $G=0$, the ground state is the closed-shell state with no decay width and the second pair-correlated state is a resonant state of the Fermion pair $S_{9}^{\dagger}$ with the half width $\Gamma / 2=\gamma=1$. As the pairing correlations become stronger, the "width" of the ground state increases and after a critical strength of $G$ it exceeds the width of the second state which is originated in a resonant state.

How does the imaginary part of the ground-state en-

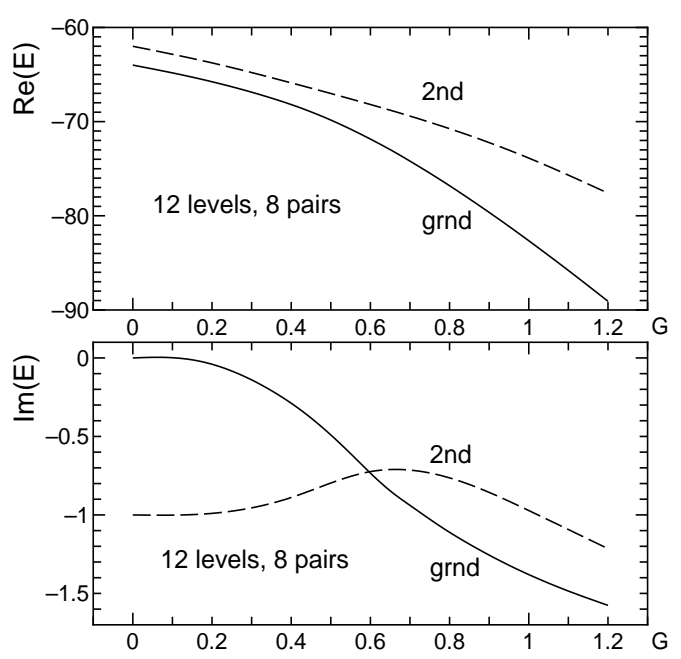

FIG. 1: Energies of the ground and next pair-correlated states of the $N=8$ system when $\gamma / 2=0.5$. Variations of real and imaginary parts depending on $G$ are illustrated. ergy $\operatorname{Im}(E)$ depend on the parameter $\gamma$ ? The upper graph of Fig. 2 illustrates $\operatorname{Im}(E)$ as a function of $\gamma / 2$, in case of $N=8$ and $G=0.3$. The value of $\operatorname{Im}(E)$ is proportional to $\gamma$ in a small $\gamma$ region, but reaches the top at $\gamma / 2 \approx 2$ after becoming a gentle slope around $\gamma / 2 \sim 1$. To see the structure change, we calculated the occupation probabilities $v_{\nu}^{2}$ of respective s.p. states for the ground state. The real parts of $v_{\nu}^{2}$ for the resonant s.p. states are illustrated in the lower graph of Fig. 2. This figure indicates that the occupation probabilities $\operatorname{Re}\left(v_{\nu}^{2}\right)$ cross the zero point one after another. Especially, $\operatorname{Re}\left(v_{9}^{2}\right)$ of the lowest resonant s.p. level $\nu=9$ decreases drastically as $\gamma$ increases, and becomes negative at $\gamma / 2 \approx 1.15$. The occupation probability must be positive to keep the physical meaning. If one of $\operatorname{Re}\left(v_{\nu}^{2}\right)$ is negative, the state is unphysical. We should, therefore, stop our treatment in the unphysical situation for $\gamma / 2 \gtrsim 1.15$. This model calculation suggests that the resonant s.p. states with too large widths should be removed from the space of the pairing correlations.

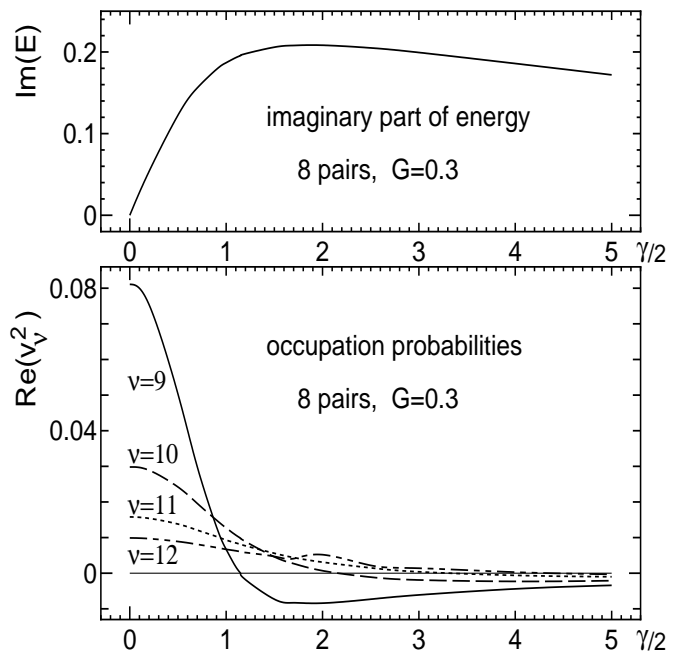

FIG. 2: The imaginary part of the ground-state energy $\operatorname{Im}(E)$ and the occupation probabilities $\operatorname{Re}\left(v_{\nu}^{2}\right)$ of the resonant s.p. levels as a function of $\gamma / 2$, in case of $N=8$ and $G=0.3$.

It should be noted that Figs. 1 and 2 give information about how the quantities vary when the pairing interactions are weakened for the resonant s.p. states or widths $\gamma$ are narrowed.

When $N=9$, the last pair occupies the resonant s.p. levels in our model. This situation is similar to the system of a core plus one pair considered in Refs. [6, 7], which discussed important roles of the resonant and continuum s.p. states in binding of the last one pair. In Fig. 3, we show the total energy of the $N=9$ system (ground state) as a function of $G$. The real part $\operatorname{Re}(E)$ is measured from the base level -64 that is the unperturbed core energy $\Sigma_{\nu=1}^{8} 2(\nu-8.5)$. If we regard the $N=8$ inner part as a core after Refs. [6, 7], the system is reduced to one pair moving in the 4 resonant s.p. levels. Its energy shown by the dash curve (a) decreases slowly as 
$G$ increases and becomes negative. The negative energy means binding (confinement) of the last pair in the system, according to Ref. 7]. The imaginary part of energy $\operatorname{Im}(E)$ remains being 1 in the truncated model (a) which has only 4 resonant s.p. levels with $\gamma / 2=0.5$.

If we take the 8 bound s.p. levels into account, the ground-state energy is very much lowered as shown by the solid curve (b) in Fig. 3. The energy crosses the zero point at much smaller $G(\approx 0.11)$ as compared with (a). Obviously, the inclusion of the bound s.p. states strengthens the correlation energy. This, however, does not mean the binding of the last pair, because the 8 pair system without the last pair also gets large binding energy. The energy difference between the 9 and 8 pair systems, which is equal to the pair decay $Q$-value (we denote it by $\left.Q_{\text {pair }}\right)$, is plotted by the dot curve $\left(\mathrm{b}^{\prime}\right)$ in Fig. 3 . The curve indicates that the $N=9$ system cannot bind the last pair in our model assuming the same potential depth for $N=8$ and $N=9$. We can say that the correlations of the last pair in the unbound s.p. states with the inner part are very important but whether the last pair is bound or not in the system may depend on complicated conditions. (It is expected that the $2 N+1=17$ system is more loosely bound due to the blocking effect than the $2 N=18$ system.) The imaginary part of energy $\operatorname{Im}(E)$ increases also in the $N=9$ system as $G$ increases, in our model without the continuum s.p. states.

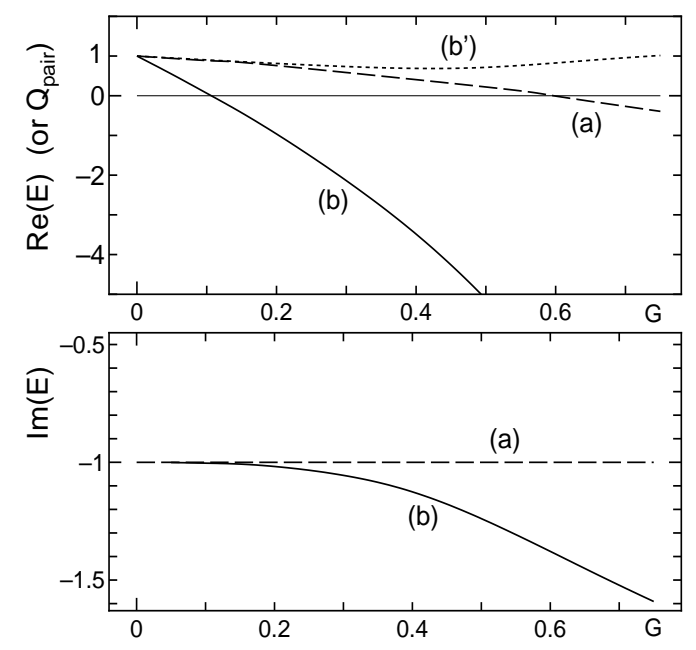

FIG. 3: Ground-state energy of the $N=9$ system $(\gamma / 2=$ 0.5): (a) neglects the bound s.p. states; (b) includes them.

The previous work [6, 7] pointed out important roles of the continuum s.p. states in the core plus one pair system. The calculations [6, 7] showed that the continuum states contribute to the binding of the last pair in the system without bound s.p. states like (a). If the continuum s.p. states are correctly treated, therefore, we can expect that the continuum states may contribute to the binding of the last pair in the system including bound s.p. states like (b) and that the imaginary part of energy $\operatorname{Im}(E)$ decreases reasonably.
TABLE I: Occupation probabilities $v_{a}^{2}$ of respective spherical orbits $a$ in the ground state of ${ }^{24} \mathrm{O}$ when $G=0.61 \mathrm{MeV}$.

\begin{tabular}{c|c|cc|cc|cc}
\hline & & \multicolumn{2}{|c|}{ neglect $\gamma_{a}$} & \multicolumn{2}{c|}{ include $\gamma_{a}$} & \multicolumn{2}{c}{ exclude $\circ$} \\
& $\varepsilon_{a}(\mathrm{MeV})$ & $v_{a}^{2}$ & $\Delta_{a}$ & $\operatorname{Re}\left(v_{a}^{2}\right)$ & $\Delta_{a}$ & $\operatorname{Re}\left(v_{a}^{2}\right)$ & $\operatorname{Re}\left(v_{a}^{2}\right)$ \\
\hline $1 f_{7 / 2}$ & $5.49-\mathrm{i} 0.69$ & 0.026 & 0.39 & 0.022 & 0.36 & 0.013 & $\circ$ \\
$2 p_{1 / 2}$ & $1.74-\mathrm{i} 3.06$ & 0.069 & 0.15 & 0.018 & 0.08 & $\circ$ & $\circ$ \\
$2 p_{3 / 2}$ & $0.45-\mathrm{i} 2.06$ & 0.110 & 0.38 & 0.047 & 0.26 & $\circ$ & $\circ$ \\
$1 d_{3 / 2}$ & -0.07 & 0.137 & 0.42 & 0.137 & 0.42 & 0.082 & 0.056 \\
\hline $2 s_{1 / 2}$ & -3.12 & 0.759 & 0.26 & 0.835 & 0.23 & 0.923 & 0.960 \\
$1 d_{5 / 2}$ & -4.54 & 0.872 & 0.61 & 0.908 & 0.53 & 0.960 & 0.978 \\
$1 p_{1 / 2}$ & -13.34 & 0.985 & 0.08 & 0.987 & 0.07 & 0.995 & 0.998 \\
$1 p_{3 / 2}$ & -16.26 & 0.989 & 0.11 & 0.991 & 0.11 & 0.997 & 0.999 \\
$1 s_{1 / 2}$ & -28.06 & 0.997 & 0.03 & 0.997 & 0.03 & 0.998 & 1.000 \\
\hline$\Delta$ & & & 2.45 & \multicolumn{5}{c}{2.09} & 1.27 & 0.73 \\
$E$ & & -189.95 & -189.29 & -187.77 & -186.91 \\
\hline
\end{tabular}

Now let us consider sixteen neutrons of ${ }^{24} \mathrm{O}$ as an example of realistic system. We neglect the continuum s.p. states, because we do not have a reliable treatment of them in our method. We generated the single-neutron basis for ${ }^{24} \mathrm{O}$ by a Woods-Saxon potential with the radius $R_{0}=1.2 A^{1 / 3} \mathrm{fm}$, the surface diffuseness $a=0.65$ $\mathrm{fm}$, the potential depth $V_{0}=-58 \mathrm{MeV}$ and the strength of the spin-orbit term $U_{\text {so }}=15 \mathrm{MeV}$ [ . The s.p. energies obtained are listed in the second column of Table I. The energies $\varepsilon_{a}$ are similar to those obtained with the Cwoik code [10], except for the very low energies of the $2 p_{3 / 2}$ and $2 p_{1 / 2}$ orbits. The resonant widths are large for $2 p_{3 / 2}$ and $2 p_{1 / 2}$. Using the s.p. energies, we solved the Richardson equations for the lowest three pair-correlated states of ${ }^{24} \mathrm{O}$, by varying the pairing force strength $G$. Though we omit figures, calculated result shows the increase of the pairing correlation energy as $G$ increases, which resembles the small $G$ region of Fig. 1. The general features shown by the model (4) are confirmed in ${ }^{24} \mathrm{O}$.

Table I lists the real parts of the occupation probabilities $v_{a}^{2}$ and pairing gap $\Delta=\Sigma_{a} \Delta_{a}=\Sigma_{a} G\left(j_{a}+\right.$ $1 / 2) v_{a} \sqrt{1-v_{a}^{2}}$, for the ground state. Here, we set $G=0.61 \mathrm{MeV}$ so that $\Delta$ is nearly the standard value $12 / \sqrt{A} \mathrm{MeV}$ when $\gamma_{a}=0$. Table I indicates that the widths $\gamma_{a}$ disturb the pairing correlations and pairs hesitate in jumping to the resonant orbits with large widths. The pairing gap $\Delta$ is $2.45 \mathrm{MeV}$ when neglecting $\gamma_{a}$, while it is $2.09 \mathrm{MeV}$ when we take account of $\gamma_{a}$. This feature agrees with that of the HF+BCS calculation [4]. It is important to notice that the resonant orbits with large widths participate little in the pairing correlations. This property is sharply revealed if the widths of $2 p_{3 / 2}$ and $2 p_{1 / 2}$ are larger. When using another parameters for the Woods-Saxon potential, we had negative values of $\operatorname{Re}\left(v_{a}^{2}\right)$ for $2 p_{3 / 2}$ and $2 p_{1 / 2}$, which corresponds to the unphysical situation pointed out in Fig. 2.

Kruppa et al. [5] argued that only resonant s.p. states with narrow widths should be included, to keep physical meaning. If we exclude the orbits $2 p_{3 / 2}$ and $2 p_{1 / 2}$ following them, the results become very different as shown 
in the seventh column of Table I. Excluding $2 p_{3 / 2}$ and $2 p_{1 / 2}$ changes the occupation probabilities $\operatorname{Re}\left(v_{a}^{2}\right)$ very much. The pairing gap $\Delta$ decreases from $2.09 \mathrm{MeV}$ to $1.27 \mathrm{MeV}$. (It is interesting to note that the values of $\operatorname{Re}\left(v_{a}^{2}\right)$ approach those of the fifth column including $2 p_{3 / 2}$ and $2 p_{1 / 2}$, if $G$ is enlarged so as to give the same gap $\Delta \approx 2.09 \mathrm{MeV}$.) The significant reduction means that the resonant s.p. states with large widths, if they are included, are little occupied but still contribute to the pairing correlations. If we neglect the last resonant s.p. orbit $1 f_{7 / 2}$ further, the pairing correlations are much weakened as shown in the last column of Table I $(\Delta=0.73 \mathrm{MeV})$. On the ground-state energy tabulated in the bottom line of Table I, whether including or excluding the resonant s.p. states $2 p_{3 / 2}$ and $2 p_{1 / 2}$ is more decisive than the effect of the resonant widths $\gamma_{a}$. Exclusion of $2 p_{3 / 2}$ and $2 p_{1 / 2}$ reduces the energy gain about $1.5 \mathrm{MeV}$ (from 189.29 to 187.77), while the resonant widths $\gamma_{a}$ reduces the energy gain $0.66 \mathrm{MeV}$ (from 189.95 to 189.29 ). The imaginary part of the ground-state energy $\operatorname{Im}(E)$ becomes very small $-0.09 \mathrm{MeV}$ from $-1.12 \mathrm{MeV}$, if the resonant orbits
$2 p_{3 / 2}$ and $2 p_{1 / 2}$ with large widths are excluded, which complies with the physical demand [5].

In conclusion, we have investigated the effects of the resonant s.p. states on the pairing correlations by an exact treatment of the pairing Hamiltonian. The present study shows that many-body correlations between bound and resonant particle pairs are important and that resonant s.p. states with too large widths should not be included in the space of the pairing correlations. We have not completed the study of the contribution from the continuum s.p. states. The interactions related to the resonant s.p. states and especially to the continuum s.p. states must be appropriately evaluated. Our approach using the Richardson equations is not adequate to this problem. It is necessary to use different pairing interactions in the next step. The conclusion that resonant s.p. states with very short life time should be excluded from the space of the pairing correlations must be investigated further in calculations which deal with the continuum s.p. states correctly.
[1] A. B. Migdal, Sov. J. Nucl. Phys. 16, 238 (1973).

[2] J. R. Bennett, J. Engel and S. Pittel, Phys. Lett. B 368, 7 (1996).

[3] J. Dobaczewski, W. Nazarewicz, T. R. Werner, J. F. Berger, C. R. Chinn and J. Dechargé, Phys. Rev C 53, 2809 (1996).

[4] N. Sandulescu, Nguyen Van Giai and R. J. Liotta, Phys. Rev C 61, 061301 (2000).

[5] A. T. Kruppa, P. H. Heenen and R. J. Liotta, Phys. Rev C 63, 044324 (2001).

[6] R. Id Betan, R. J. Liotta, N. Sandulescu and T. Vertse,
Phys. Rev. Lett. 89, 042501 (2002).

[7] N. Michel, W. Nazarewicz, M. Ploszajczak and K. Bennaceur, Phys. Rev. Lett. 89, 042502 (2002).

[8] R. W. Richardson and N.Sherman, Nucl. Phys. 52, 221 (1964); 52, 253 (1964).

[9] T. Vertse, K. F. Pal and Z. Balogh, Comput. Phys. Commun. 27, 309 (1982).

[10] S. Cwoik, J. Dudek, W. Nazarewicz, J. Skalski and T. Werner, Comput. Phys. Commun. 46, 379 (1987). 